\title{
Indeterminate/moderate IgG avidity during HCMV infection: comparison of methods
}

\author{
Massimo De Paschale, Alessia Paganini, Maria Teresa Manco, Laura Marinoni, Luigi Bertinotti, Carlo Agrappi, \\ Paola Mirri, Arianna Gatti, Cristina Rescaldani, Pierangelo Clerici \\ U.O. Microbiologia \\ A.O. Ospedale Civile di Legnano (Mi)
}

Key words: IgG avidity; anti-HCMV antibodies, HCMV infection

Avidità indeterminata/moderata delle IgG in corso d'infezione da HCMV: confronto tra metodiche

SUMMARY

Background. The IgG avidity test is usually used for differentiating between primary and non-primary HCMV infection within 3 months. Weak avidity is highly suggestive of a primary infection, while high avidity tends to exclude it. An indeterminate or moderate avidity, however, does not allow a clear dating. Since there are several avidity tests with different performances, those tests that are able to minimize the results with indeterminate/moderate avidity are particularly useful.

Objectives. The aim of our work was to evaluate the results obtained with two IgG avidity tests in IgG and IgM anti-HCMV positive patients. Study Design. II 3 anti-HCMV IgG and IgM positive samples were tested with Enzime Linked Fluorescent Assay (ELFA) and Chemiluminescence Immuno Assay (CLIA) IgG avidity test.

Results. 2 I samples (I8.6\%), 50 (44.2\%) and $42(37.2 \%)$ with ELFA and 53 samples (46.9\%), 10 (8.8\%) and 50 (44.2\%) with CLIA were found to have respectively low, indeterminate/moderate and high avidity. Of the 50 ELFA indeterminate avidity samples, $32(64 \%)$, $10(20 \%)$ and 8 (16\%) were found to have respectively low, moderate and high CLIA.avidity. For II cases of the 32 ELFA indeterminate avidity and CLIA low avidity, there were previous data showing a seroconversion within three months. In a case of the 8 ELFA indeterminate avidity and CLIA high avidity, there were, instead, previous data of $\lg G$ and $\lg M$ positivity already four months earlier.

Conclusions. It appears that the CLIA test for $\lg G$ avidity is more effective than ELFA for dating HCMV infection.

\section{INTRODUZIONE}

Il citomegalovirus umano (HCMV) è un virus ubiquitario responsabile, nell'ospite immunocompetente, di infezioni generalmente asintomatiche e, solo in pochi casi, di mononucleosi e epatite (9) soprattutto a carico di pazienti immunocompromessi. Particolarmente importante è l'infezione congenita con manifestazioni cliniche che vanno da forme asintomatiche $(90 \%$ dei casi) a gravi danni al feto fino, in rari casi, alla morte per aborto $(6,7,19)$. In seguito alla latenza che segue un'infezione primaria possono esserci periodiche riattivazioni per cui la trasmissione in utero può avvenire sia durante un'infezione primaria che durante le riattivazioni (4) anche se il rischio di infezione congenita è più alta nelle infezioni primarie rispetto ai casi di riattivazione o di reinfezione con altri ceppi $(4,6,7,20)$. Per la diagnosi sierologica di infezione da HCMV si utilizzano test per la ricerca di anticorpi anti-HCMV di classe G e M. L'utilizzo delle IgM, presenti nelle infezioni primarie (10), ha, però alcuni limiti in quanto, in alcuni casi, le IgM possono essere ancora positive, anche se generalmente a livelli bassi, per più di 12 mesi (18). Le IgM possono, inoltre, essere prodotte anche durante le riattivazioni o reinfezioni soprattutto nei pazienti immunocompromessi $(14,16)$ o, in corso di infezione primaria da EBV, come conseguenza dell'attivazione policlonale dei linfociti B da parte dell'EBV (5). Di conseguenza le IgM, una volta confermate, possono rilevare differenti situazioni come infezione primaria acuta, fase convalescente di infezione primaria o persistenza di IgM soprattutto se i livelli delle IgM sono bassi (16). Risulta determinante, quindi, utilizzare altri test per distinguere tra infezione primaria e non primaria non solo in gravidanza per identificare infezioni a rischio di trasmissione al feto, ma anche, in pazienti con sintomatologia clinica evidente, per porre una diagnosi differenziale con altre infezioni a sintomatologia simile. In tali situazioni è stato ampiamente utilizzato il test dell'avidità delle $\operatorname{IgG}(2,8,11,12,16)$.

Le IgG con bassa avidità sono prodotte inizialmente durante il primo mese dell'infezione e man mano che passa il tempo si producono IgG ad alta avidità che risultano essere le sole evidenziabili nei casi di infezioni pregresse o ricorrenti.
Utilizzando un test capace di distinguere tra IgG a bassa o ad alta avidità è possibile, quindi, caratterizzare un'infezione. La presenza di alti livelli di IgM e debole avidità, è altamente suggestiva di una infezione contratta da meno di 3 mesi, mentre un'alta avidità tenderebbe ad escluderla (15). I test attualmente in commercio utilizzano un indice di avidità che esprime la percentuale di IgG legate all'antigene in seguito al trattamento con una sostanza denaturante. I risultati sono espressi numericamente ed interpretati come avidità alta, bassa o indeterminata o moderata a secondo dei kit in uso. In caso di avidità indeterminata non è possibile distinguere tra una infezione recente e pregressa. Anche un'avidità moderata non esclude la possibilità di un'infezione recente, ma può indicare un'infezione pregressa con maturazione non completa dell'avidità delle IgG, anche se studi recenti riportano come un'infezione primaria possa essere diagnosticata dalla presenza di un'avidità bassa/moderata che persiste per circa 18-20 settimane dopo l'infezione primaria (13).

Poiché in commercio esistono vari test proposti da diverse aziende per l'avidità delle IgG con performance diverse (3), i risultati ottenuti, soprattutto in termini di ampiezza del range numerico che intercorre tra un'avidità bassa e alta, sono diversi e questo comporta differenze nelle conclusioni che possono essere tratte.

Scopo del nostro lavoro è stato quello di valutare le risposte con due test per l'avidità delle IgG in pazienti positivi per la ricerca delle IgM anti-HCMV.

\section{MATERIALI E METODI}

Tra il 2005 e il 2007 all’U.O. di Microbiologia dell’Ospedale Civile di Legnano sono giunti 6990 campioni di altrettanti soggetti, di cui 4173 (1625 uomini e 2548 donne, età media 39.2 anni; range 1 -99) erano pazienti prelevati per un controllo clinico, mentre 2817 erano donne gravide (età media 32.3 anni; range 15-46 anni) sottoposte allo screening sierologico in gravidanza.

A tutti sono stati ricercati gli anticorpi anti-HCMV IgG e IgM (ETI-CYTOK-G-PLUS e ETI-CYTOK-M reverse PLUS, DiaSorin, Saluggia, Italia). Sui campioni risultati IgM ELISA 
positivi, è stata determinata l'avidità delle IgG, ove possibile, con metodica ELFA (Enzyme-Linked Fluorescent Assay: VIDAS CMV IgG Avidity, bioMérieux, Lione, Francia).

Retrospettivamente, in base alla disponibilità di siero congelato, è stata determinata l'avidità delle IgG con metodica CLIA (Chemiluminescenza Immuno Assay: LIAISON CMV IgG Avidity, DiaSorin Saluggia, Italia).

Nel test ELFA, l'avidità era considerata debole se l'indice era $<0.200$, forte se l'indice era $\geq 0.800$ e indeterminata se l'indice era $\geq 0.200 \mathrm{ma}<0.800$ (1).

Nel test CLIA, l'avidità era considerata bassa se l'indice era $<0.200$, moderata se l'indice era compreso tra 0.200 e 0.300 , ed elevata se l'indice era $\geq 0.300$ (17).

Per entrambi i test, in caso di avidità indeterminata o moderata da brochure nel kit non è possibile distinguere tra infezione recente e pregressa.

Per l'analisi statistica sono stati usati il test chi quadro e il test esatto di Fisher.

\section{RISULTATI}

Dei 6990 campioni, 148 (2.1\%) erano risultati positivi alla ricerca di entrambi gli anticorpi anti-HCMV IgG e IgM. Con il test ELFA per l'avidità delle IgG, 28 (18.9\%) erano risultati avere avidità debole, 64 (43.2\%) avidità forte e 56 (37.8\%) avidità indeterminata. In base alla disponibilità di siero congelato sono stati selezionati 113 campioni: 21 (18.6\%) con avidità debole $50(44.2 \%)$ con avidità indeterminata e 42 (37.2\%) con avidità forte al test ELFA. Dei 113 campioni in esame, 87 appartenevano ad altrettanti pazienti (56 uomini e 31 donne) prelevati per un controllo clinico e 26 a donne in gravidanza. Di queste, 23 erano al primo trimestre di gravidanza, due al secondo e una al terzo.

Al test CLIA dei 113 campioni, 53 (46.9\%) sono risultati con avidità debole, $10(8.8 \%)$ con avidità moderata e 50 (44.2\%) con avidità forte. I risultati suddivisi per pazienti e donne in gravidanza sono riportati in Tabella 1. La differenza tra le percentuali ottenute per i campioni con avidità indeterminata/moderata con i due metodi è statisticamente significativa. Il confronto tra i due metodi è riportato in Tabella 2. In particolare tutti i campioni risultati deboli al test ELFA sono risultati deboli al test CLIA e tutti i campioni forti al test ELFA sono risultati forti anche al test CLIA.

Considerando i 50 campioni con avidità indeterminata al test ELFA, con il test CLIA 32 campioni (64.0\%) sono risultati deboli, 10 (20.0\%) moderati e 8 (16.0\%) forti (Tabella 3). Per 11 casi (tutti pazienti) dei 32 campioni indeterminati al test ELFA e deboli al test CLIA si avevano dati precedenti indicanti una sieroconversione avvenuta entro tre mesi dal prelievo del campione in studio. In un caso degli 8 campioni indeterminati all'ELFA e forti al CLIA si aveva, invece, un dato di positività per IgG e IgM già quattro mesi prima.

Infine, per due casi (di cui una donna gravida) tra i 10 con avidità indeterminata/moderata con entrambi i test, si avevano dati precedenti indicanti una sieroconversione entro tre mesi.
Tabella 2. Confronto tra due test per l'avidità delle IgG (ELFA e CLIA) su campioni risultati positivi alla ricerca di anticorpi anti-HCMV IgG e IgM.

\begin{tabular}{lcccc}
\hline \multicolumn{5}{c}{ Avidità IgG } \\
\hline ELFA & \multicolumn{4}{c}{ CLIA } \\
\hline & Debole & Moderata & Forte & Totale \\
\hline Debole & 21 & 0 & 0 & 21 \\
\hline Indeterminata & 32 & 10 & 8 & 50 \\
\hline Forte & 0 & 0 & 42 & 42 \\
\hline Totale & 53 & 10 & 50 & 113 \\
\hline
\end{tabular}

Tabella 3. Risultati con il test per l'avidità lgG in CLIA di 50 campioni con avidità indeterminata al test ELFA.

\begin{tabular}{lcccc}
\hline Casistica & $\mathbf{N}^{\circ}$ & \multicolumn{4}{c}{ Avidità IgG (CLIA) } \\
\hline Pazienti & 37 & Debole & Moderata & Forte \\
\hline Gravide & 13 & $5(33 \%)$ & $5(13.5 \%)$ & $5(13.5 \%)$ \\
\hline Totale & 50 & $32(64.0 \%)$ & $5(38.5 \%)$ & $3(23.1 \%)$ \\
\hline
\end{tabular}

\section{DISCUSSIONE}

La gravità dell'infezione congenita da HCMV richiede una precisa diagnosi di infezione acuta in gravidanza. Essendo l'infezione spesso asintomatica i test di laboratorio sono gli unici a dare indicazione di una possibile infezione primaria. Tra i test disponibili, la ricerca delle IgM è ampiamente utilizzata a questo scopo, ma la loro possibile permanenza prolungata, la loro produzione anche in caso di reinfezioni e riattivazioni o possibili cross-reazioni con altri virus ne limitano l'utilizzo come marcatore di infezione primaria. Per individuare quest'ultima, è utilizzato il test per l'avidità delle IgG che permette, nonostante qualche limite in relazione alla maturazione individuale delle IgG, di distinguere una infezione primaria da HCMV contratta da meno di 3 mesi da una non primaria. Questo è particolarmente importante per le donne in gravidanza che si sottopongono ad uno screening sierologico nel primo trimestre. Una debole avidità è altamente suggestiva di una infezione contratta da meno di 3 mesi e, quindi, prima del concepimento, mentre un'alta avidità la escluderebbe.

La situazione appare più problematica quando l'avidità appare indeterminata/moderata in quanto non è possibile distinguere tra infezione recente e pregressa e, per poter chiarire la situazione, bisogna ricorrere ad altri metodi e/o ad un follow up anticorpale.

È auspicabile, quindi, mantenere questa situazione di indeterminatezza nel minor numero di casi possibili per cui utilizzare test che siano in grado di minimizzare tali numeri è di particolare utilità. Con il test ELFA il 44.2\% dei campioni risultava avere avidità indeterminata a fronte dell' $8.8 \%$ di avidità moderata con il test in CLIA.

In particolare tra le donne gravide, il test CLIA riusciva a distinguere tra infezione contratta entro tre mesi o più di tre mesi in più della metà dei casi che erano risultati indeterminati al test ELFA.

Poiché si aveva a disposizione, in alcuni tra i casi indeterminati al test ELFA e deboli al test CLIA, dati indicanti una seroconversione recente (meno di tre mesi), in questi

Tabella I. Risultati con il test ELFA e CLIA per l'avidità delle lgG di II 3 campioni risultati positivi alla ricerca di anticorpi anti-HCMV IgG e IgM,

\begin{tabular}{|c|c|c|c|c|c|}
\hline \multirow[t]{2}{*}{ Casistica } & \multirow[t]{2}{*}{$\mathbf{N}^{\circ}$} & \multirow[t]{2}{*}{ Test } & \multicolumn{3}{|c|}{ Avidità IgG } \\
\hline & & & Debole & Indeterminata/Moderata & Forte \\
\hline \multirow[t]{3}{*}{ Pazienti } & 87 & ELFA & $20(23 \%)$ & $37(42.5 \%)$ & $30(34.5 \%)$ \\
\hline & & CLIA & $47(54 \%)$ & $5(5.7 \%)$ & $35(40.2 \%)$ \\
\hline & & $\bar{p}$ & $<0.01$ & $<0.01$ & NS \\
\hline \multirow[t]{3}{*}{ Gravide } & 26 & ELFA & $\mathrm{I}(3.8 \%)$ & $13(50 \%)$ & $12(46.2 \%)$ \\
\hline & & CLIA & $6(23.1 \%)$ & $5(19.2 \%)$ & $15(57.7 \%)$ \\
\hline & & $P$ & NS & $<0.05$ & NS \\
\hline \multirow[t]{3}{*}{ Totale } & 113 & ELFA & $21(18.6 \%)$ & $50(44.2 \%)$ & $42(37.2 \%)$ \\
\hline & & CLIA & $53(46.9 \%)$ & $10(8.8 \%)$ & $50(44.2 \%)$ \\
\hline & & $\mathrm{p}$ & $<0.01$ & $<0.01$ & NS \\
\hline
\end{tabular}

NS = non significativo 
casi il test CLIA aveva correttamente individuato un'infezione acuta.

In conclusione il test CLIA sembra essere maggiormente in grado di datare l'infezione. Il test ELFA, invece, presentando un range numerico molto ampio, è più prudente nell'individuare un'infezione acuta, ma il suo utilizzo risulta poco incisivo soprattutto in caso di donne in gravidanza.

\section{BIBLIOGRAFIA}

1. Baccard-Longere M, Freymuth F, Cointe D, Seigneurin JM, GrangeotKeros L. Multicenter evaluation of a rapid and convenient method for determination of Cytomegalovirus immunoglobulin G avidity. Clin Diagn Lab Immunol 2001; 8: 429-431.

2. Blackburn NK, Besselaar TG, Schoub BD, O’Connell KF. Differentiation of primary cytomegalovirus infection from reactivation using the urea denaturation test for measuring antibody avidity. J Med Virol 1991; 33: 6-9.

3. Bodéus M, Beulné D, Goubau P. Ability of three IgG-avidity assays to exclude recent Cytomegalovirus infection. Eur J Clin Microbiol Infect Dis 2001; 20: 248-252.

4. Boppana SB, Rivera LB, Fowler KB, Mach M, Britt WJ. Intrauterine transmission of cytomegalovirus to infants of women with preconceptional immunity. N Engl J Med 2001; 344: 1366-1371.

5. Deyi YM, Goubau P, Bodéus M. False-positive IgM antibody tests for cytomegalovirus in patients with acute Epstein-Barr virus infection. Eur $J$ Clin Microbiol Infect Dis 2000; 19: 557-560.

6. Fowler KB, Stagno S, Pass RF, Britt WJ, Boll TJ, Alford CA. The outcome of congenital cytomegalovirus infection in relation to maternal antibody status. $N$ Engl J Med 1992; 326: 663-667.

7. Fowler KB, Stagno S, Pass RF. Maternal immunity and prevention of congenital cytomegalovirus infection. JAMA 2003; 289: 1008-1011.

8. Grangeot-Keros L, Mayaux MJ, Lebon P, et al. Value of cytomegalovirus (CMV) IgG avidity index for the diagnosis of primary CMV infection in pregnant women. J Infect Dis 1997; 175: 944-946.

9. Griffiths PD. Strategies to prevent CMV infection in the neonate. Semin
Neonatol 2002; 7: 293-299.

10. Kenneson A, Cannon MJ. Review and meta-analysis of epidemiology of congenital cytomegalovirus (CMV) infection. Rev Med Virol 2007; 17: 253-276.

11. Lazzarotto T, Spezzacatena P, Pradelli P, Abate DA, Varani S, Landini MP. Avidity of immunoglobulin $\mathrm{G}$ directed against human cytomagalovirus during primary and secondary infections in immunocompetent and immunocompromised subjects. Clin Diagn Lab Immunol 1997; 4: 469473.

12. Lazzarotto T, Spezzacatena P, Pradelli P, et al. Cytomegalovirus infection in pregnancy: a still complicated diagnostic problem. Intervirology 1998: 41: 149-157.

13. Lazzarotto T, Guerra B, Lanari M, Gabrielli L, Landini MP. New advances in the diagnosis of congenital cytomegalovirus infection. J Clin Virol 2008; 41: 192-197.

14. Nielsen SL, Sørensen I, Andersen HK. Kinetics of specific immunoglobulins $\mathrm{M}, \mathrm{E}, \mathrm{A}$, and $\mathrm{G}$ in congenital, primary, and secondary cytomegalovirus infection studied by antibody-capture enzyme-linked immunosorbent assay. J Clin Microbiol 1988; 26: 654-661.

15. Revello MG, Gerna G. Diagnosis and implications of human cytomegalovirus infection in pregnancy. Fet Matern Med Rev 1999; 11 $117-134$

16. Revello MG, Gerna G. Diagnosis and management of human Cytomagalovirus infection in the mother, fetus and newborn infant. Clin Microbiol Rev 2002; 15: 680-715.

17. Revello MG, Gorini G, Gerna G. Clinical evaluation of a chemiluminescence immunoassay for determination of immunoglobulin $G$ avidity to human Cytomegalovirus. Clin Diagn Lab Immunol 2004; 11: 801-805.

18. Revello MG, Percivalle E, Zannino M, Rossi V, Gerna G. Development and evaluation of a capture ELISA for IgM antibody to the human cytomegalovirus major DNA binding protein. J Virol Methods 1991; 35: 315-329.

19. Stagno S, Pass RF, Cloud G, et al. Primary cytomegalovirus infection in pregnancy : incidence, transmission to fetus and clinical outcome. JAMA 1986; 256:1904-8.

20. Stagno S, Pass RF, Dworsky ME, et al. Congenital cytomegalovirus infection. The relative importance of primary and recurrent maternal infection. $N$ Eng J Med 1982; 306: 945-949. 